\title{
Evacuation of Spontaneous Thalamic and Intraventricular Hemorrhage under the Operating Microscope Improves Mortality Compared with External Ventricular Drainage
}

-Mini-Cranioctomy for Thalamic and Ventricular Hemorrhage

\author{
Tomonori Tamaki $^{1}$, Yoji Node ${ }^{1}$, Akira Teramoto ${ }^{2}$ \\ ${ }^{1}$ Nippon Medical School Tamanagayama Hospital Department of Neurosurgery, Tokyo, Japan \\ ${ }^{2}$ Nippon Medical School Department of Neurosurgery, Tokyo, Japan \\ E-mail:tamakito@nms.ac.jp \\ Received June 21, 2011; revised July 14, 2011; accepted July 15, 2011
}

\begin{abstract}
We performed direct hematoma evacuation of thalamic and intraventricular hemorrhage using mini-craniotomy in recent years. The present study evaluated the outcome and complications in 18 patients with spontaneous thalamic and intraventricular hemorrhage treated by mini-craniotomy hematoma evacuation with external ventricular drainage and 24 patients treated by only external ventricular drainage. Patients treated by mini-craniotomy were less likely to require days of ventricular drainage settlement, had a less suffering meningitis, had good hematoma evacuation rate and had a less mortality rate compared with those undergoing only external ventricular drainage. Frontal mini-craniotomy microscope operation is a simple and effective method for hematoma evacuation that causes fewer complications.
\end{abstract}

Keywords: Thalamic Hemorrhage, Intraventricular Hemorrhage, Hydrocephalus

\section{Introduction}

When thalamic hemorrhage (Th Hx) is accompanied by severe intraventricular hemorrhage (IVH), the prognosis is poor [1-5]. Obstruction of the circulation of cerebrospinal fluid (CSF) result in neurological deterioration. The goal of treatment should be to evacuate IVH in the acute stage. Th $\mathrm{Hx}$ and IVH usually treated by external ventricular drainage (EVD) for obstructive hydrocephalus[1]. However, drainage occlusion, meningitis and residual hematoma are often troublesome in actual clinical practice. Several other opinions for Th $\mathrm{Hx}$ with IVH were reported to be CT-guided stereotactic hematoma aspiration, neuroendoscopic hematoma evacuation, and EVD with fibrinolysis [6-9]. For such severe cases, we performed direct hematoma evacuation by frontal mini-craniotomy transcortical approach in recent years [2]. The present study evaluated the outcome, mortality rate and complications in eighteen patients with spontaneous Th $\mathrm{Hx}$ and IVH treated by frontal mini-craniotomy hematoma evacuation with EVD and twenty-four pa- tients treated by only EVD.

\section{Methods}

Forty-two consecutive patients, including 25 men and 17 women aged 40 to 78 years (mean 60.6 years), underwent surgery for Th Hx with IVH between 1999 and 2010. The diagnosis was based on computed tomography (CT) findings. Some patients also underwent angiography or magnetic resonance (MR) imaging to further assess the cause of the hemorrhage, but no vascular anomalies or tumors were identified. The patients were randomly allocated to hematoma evacuation via frontal mini-craniotomy (18 patients) or EVD (24 patients). We compared the two groups with respect to age, sex, preoperative level of consciousness (Glasgow coma scale), location of the hematoma (right/left), hematoma volume (standard computerized volumetric analysis), interval from onset to operation (hours), hematoma evacuation rate [preoperative hematoma volume-postoperative hematoma volume)/preoperative hematoma $\times 100: \%$ ], ven- 
tricular drainage period (days), occurrence of meningitis, outcome at 30 days from the onset (modified Rankin Scale: 4/5/6), occurrence of epilepsy after surgery, and admission period (days) [3,4]. For statistical analysis, categorical variables were assessed by using the chi-square test and mean values of quantitative variables were compared by unpaired t-test. A probability value of less than 0.05 was considered significant. The method of hematoma evacuation was frontal mini-craniotomy with the trans-cortical approach. Hematoma evacuation was performed under general anesthesia with the patient in the supine position. Craniotomy was performed ipsilateral to the Th Hx (Figure 1). First, the frontal horn of the lateral ventricle was approached trans-cortically and hematoma was evacuated (Figure 2(a)). Then the third ventricle was approached via the foramen of Monro and IVH was evacuated (Figure 2(b)). Next, the contralateral lateral ventricle was approached via the septum pellucidum (Figure 2(c)). Finally, Th Hx was removed and an EVD tube was placed under the operating microscope (Figure $3,4)$.

\section{Results}

There were no significant differences between the two groups with respect to age, sex, preoperative level of consciousness, hematoma volume, interval from onset to operation, location of the hematoma, and postoperative occurrence of epilepsy. There was also no significant

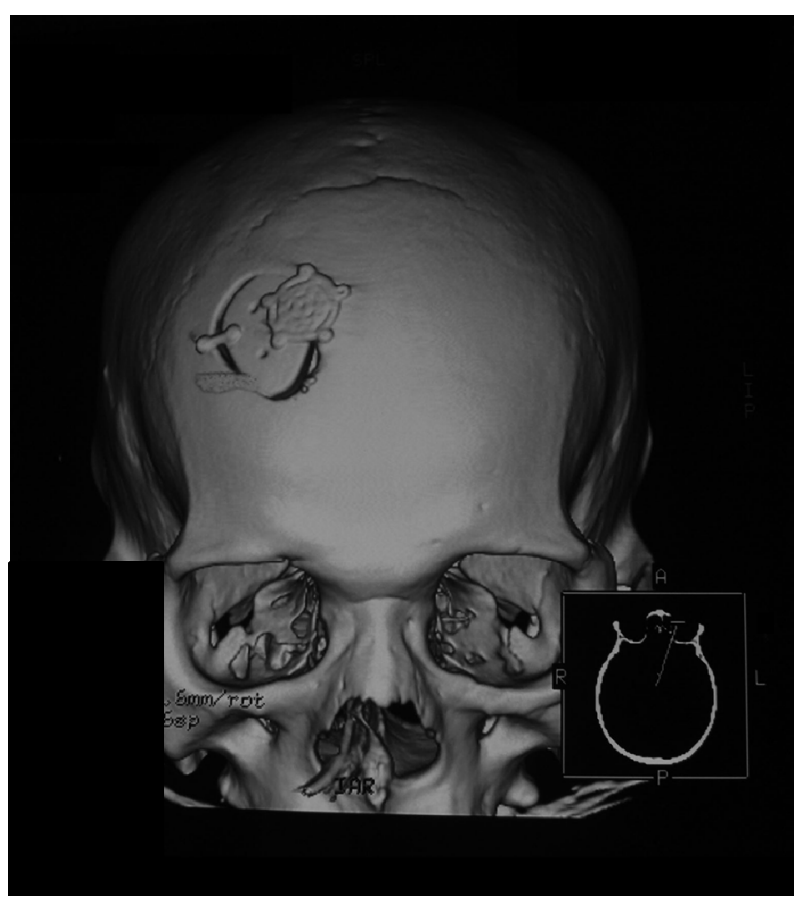

Figure 1. Three-dimensional computed tomography scan showing the mini-craniotomy at the frontal region.
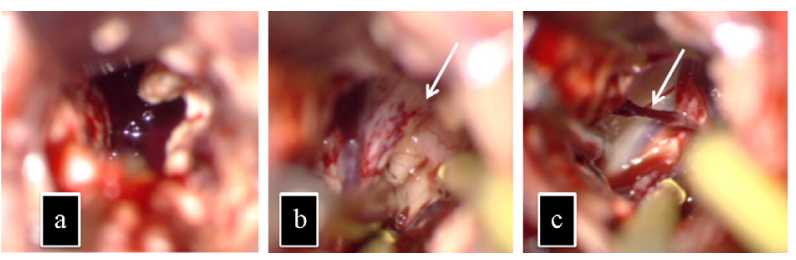

Figure 2. Intraoperative views. (a) After corticotomy of the right frontal lobe, the right lateral ventricle was fully packed with hematoma. (b) After removal of the hematoma in the third ventricle, the structure of third ventricle floor (arrow) were detected. (c) After removal of the hematoma in the left lateral ventricle, the septal vein (arrow) and injured pellucid septum were detected.

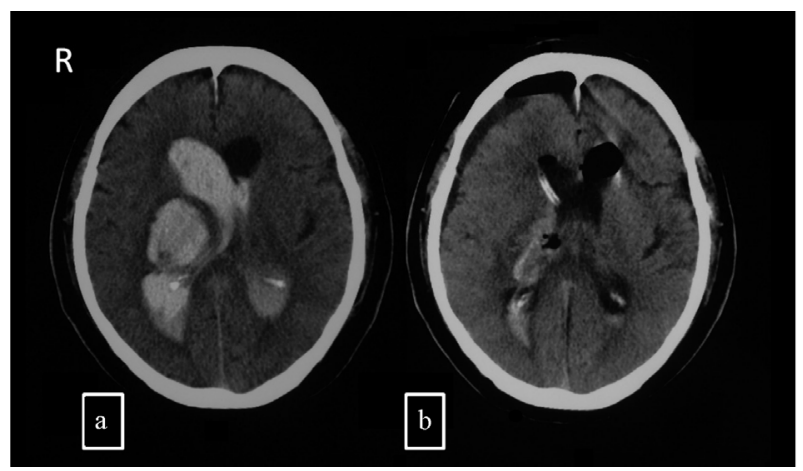

Figure 3. Frontal mini-craniotomy for the evacuation of spontaneous right thalamic and intraventricular hematoma in a 70-year-old male patient. (a) Computed tomography scan showing thalamic and intraventricular hematoma. (b) Computed tomography scan showing adequate removal of the hematoma after operation.

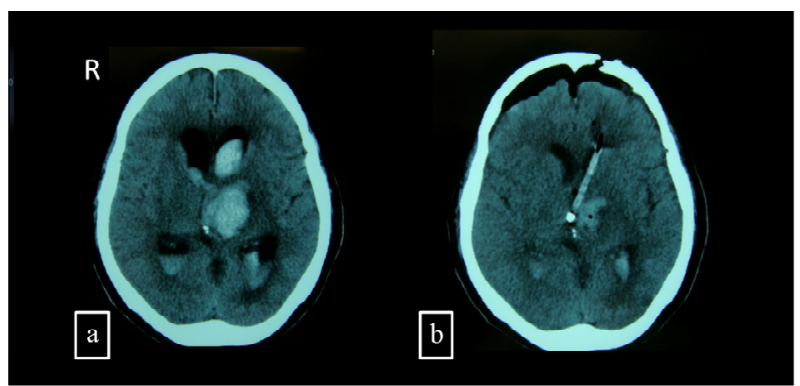

Figure 4. Frontal mini-craniotomy for the evacuation of spontaneous left thalamic and intraventricular hematoma in a 72-year-old female patient. (a) Preoperative Computed tomography scan showing thalamic and intraventricular hematoma. (b) Computed tomography scan showing adequate removal of the hematoma after mini-craniotomy.

difference of the postoperative outcome. However, patients treated by frontal mini-craniotomy with EVD were less likely to require long-term ventricular drainage and they had less meningitis, a good hematoma evacuation rate, and a lower mortality rate (mini-craniotomy: 11\% vs. EVD: 27\%) compared with those undergoing EVD alone (Table 1). 
Table 1. Clinical comparison of procedure.

\begin{tabular}{lccc}
\hline & Mini-craniotomy & Ventricular drainage & $\mathrm{p}$ Value \\
\hline Age (yrs.) & $59.6 \pm 11.9$ & $61.5 \pm 13.2$ & 0.32 \\
Sex (Male/Female) & $11 / 7$ & $14 / 8$ & 0.63 \\
Preoperative consciousness & & & \\
Level (Glasgow coma scale) & $8.1 \pm 3.2$ & $7.8 \pm 3.1$ & 0.46 \\
Location of hematoma (Rt./Lt.) & $7 / 11$ & $10 / 12$ & 0.49 \\
Hematoma volume(ml) & $59.7 \pm 12.1$ & $54.8 \pm 13.3$ & 0.52 \\
Interval from onset to operation (hrs) & $15.9 \pm 11.3$ & $10.5 \pm 6.8$ & 0.087 \\
Hematoma evacuation rate (\%) & $68.6 \pm 15.0$ & $12.7 \pm 9.8$ & 0.0003 \\
Ventricular drainage period (days) & $2.8 \pm 0.9$ & $6.1 \pm 1.8$ & 0.008 \\
Meningitis (cases) & 3 & 8 & 0.007 \\
Outcome (GOS: 4/5/6) & $6 / 10 / 2$ & $5 / 13 / 6$ & 0.26 \\
Epilepsy after operation (cases) & 2 & 1 & 0.43 \\
Mortality rate (\%) & 11 & 27 & \\
\hline
\end{tabular}

Data presented mean \pm S.D. (standard deviation); yrs: years; Rt: right; Lt: left; hrs: hours; GOS: Glasgow outcome scale

\section{Discussion}

IVH is a strong and independent predictor of a poor prognosis in patients with spontaneous intracerebral hemorrhage [1]. There is a clear rationale for the benefit of rapid removal of IVH [5,6]. However, EVD alone has little effect on hematoma clearance, because the catheter frequently becomes obstructed by blood. The standard treatment of Th Hx with IVH has been EVD. Several other opinions for Th Hx with IVH were reported to be CT-guided stereotactic hematoma aspiration, neuroendoscopic hematoma evacuation, and EVD with fibrinolysis. CT-guided stereotactic surgery has been recommended for partial evacuation of the hematoma [7]. The main advantage of CT-guided stereotactic surgery is that it can be done under local anesthesia. However, use of this technique is restricted in the acute stage, because direct hemostasis and confirmation of the source of bleeding cannot be confirmed. The rebleeding rate is $2.9 \%$ if stereotactic aspiration is done within 5 - 48 hours of the onset of hemorrhage [7]. Use of neuroendoscopy has recently been increasing for hematoma evacuation [8]. However, the surgeon must be skilled at using the neuroendoscope and few facilities have neuroendoscopic systems. Several authors have recommended intraventricular fibrinolysis (IVF) with EVD [9]. However, tissue plasminogen activator and urokinase are not used intracranially in Japan. Frontal mini-craniotomy for hematoma evacuation only needs an operating microscope and there are no specific techniques or devices. The main advantage of our method was achieving good evacuation of Th
Hx and IVH at the same time. We could perform direct hemostasis for bleeding vessels under the microscope. Our method needs a small corticotomy, so there could be criticism about cortical injury by the operation. However, the size of the corticotomy is only $2.0 \mathrm{~cm}$, which is not so different from the sheath of a neuroendoscope. There was no statistically significant difference of postoperative epilepsy between the mini-craniotomy and EVD groups [10].

\section{Conclusions}

Frontal mini-craniotomy for hematoma evacuation achieved a lower mortality rate compared with patients undergoing EVD alone. However, our method could not improve the functional outcome.

\section{References}

[1] N. J. Naff, "Intraventricular Hemorrhage in Adults," Curr eat Treatment Options in Neurology, Vol. 1, No. 3, 1999, pp. $173-178$. doi:10.1007/s11940-999-0001-0

[2] T, Tamaki and Y. Node, "Frontal Mini-Craniotomy for Evacuation of Spontaneous Thalamic and Intraventricular Hemorrhage," Surg Cereb Stroke (Japanese), Vol. 38, No. 3, 2010, pp. 181-185. doi:10.2335/scs.38.181

[3] L. Steiner, U. Bergvall and N. Zwetnow, "Quantitative Estimation of Intracerebral and Intraventricular Hematoma by Computer Tomography," Acta Radiologica Suppl, Vol. 346, 1975, pp. 143-154.

[4] J. C. Van Swieten, P. J. Koudstaal, M. C. Visser, H. J. 
Schouten and J. Van Gijn, "Interobserver Agreement for the Assessment of Handicap in Stroke Patients," Stroke, Vol. 19, No. 19, 1988, pp. 604-607. doi:10.1161/01.STR.19.5.604

[5] G. Xi, F. K. Richrd and T. H. Julian, "Mechanism of Brain Injury after Intracerebral Haemorrhage," The Lancet Neurology, Vol. 5, No. 1, 2006, pp. 53-63. doi:10.1016/S1474-4422(05)70283-0

[6] N. J. Naff, M. A. Williams, D. Rigamonti, P. M. Keyl and D. F. Hanley, "Blood Clot Resolution in Human Cerebrospinal Fluid: Evidence of First-Order Kinetics," Neurosurgery, Vol. 49, No. 3, 2001, pp. 614-619.

[7] D. Tyler and G. Mandybur, "Interventional Mri-Guided Stereotactic Aspiration of Acute/Subacute Intracerebral Hematomas," Stereotact Funct Neurosurg, Vol. 72, No. 2-4, 1999, pp. 129-135. doi:10.1159/000029712
[8] H. Hamada, N. Hayashi, M. Kurimoto, K. Umemura, S. Nagai, K. Kurosaki, N. Kuwayama and S. Endo, "Neuroendoscopic Removal of Intraventricular Hemorrhage Combined with Hydrocephalus," Minim Invasive Neurosurg, Vol. 51, No. 6, No.7, 2008, pp. 345-349. doi:10.1055/s-0028-1085449

[9] T. Morgan, M. Zuccarello, R. Narayan, P. Keyl, K. Lane and D. Hanley, "Preliminary Findings of the Minimally-Invasive Surgery Plus Rtpa for Intracerebral Hemorrhage Evacuation (Mistie) Clinical Trial,"Cerebral Hemorrhage, Vol. 105, No. 5, 2008, pp. 147-151. doi:10.1007/978-3-211-09469-3_30

[10] R. Gilad, M. Boaz, R. Dabby, M. Sadeh and Y. Lampl, "Are Post Intracerebral Hemorrhage Seizures Prevented by Anti-Epileptic Treatment?" 2011. http://www.ncbi.nlm.nih.gov/pubmed/21632213. 